\title{
Dose-enhanced combined priming regimens for refractory acute myeloid leukemia and middle- and-high-risk myelodysplastic syndrome: a single- center, retrospective cohort study
}

\author{
This article was published in the following Dove Press journal: \\ OncoTargets and Therapy \\ 20 June 2016 \\ Number of times this article has been viewed
}

\author{
Xiaorong $\mathrm{Ma}$ \\ Jin Wang \\ Yan Xu \\ Wanggang Zhang \\ Jie Liu \\ Xingmei Cao \\ Aili $\mathrm{He}$ \\ Fangxia Wang \\ Liufang Gu \\ Bo Lei \\ Jianli Wang
}

Department of Hematology, The Second Affiliated Hospital of Xi'an Jiaotong University, Xi'an, People's Republic of China
Correspondence: Xiaorong Ma Department of Hematology, The Second Affiliated Hospital of Xi'an Jiaotong University, No 157, Xiwu Road, Xi'an 710004, Shaanxi Province, People's Republic of China

Email xrma225@sina.com
Objective: To assess chemotherapeutic regimens for refractory acute myeloid leukemia (AML) and middle-and-high-risk myelodysplastic syndrome (MDS).

Methods: Between 2004 and 2014, 44 patients with refractory AML and 36 patients with MDS were treated with new priming regimens (CHAG, CHTG, CHMG, or CTMG), and 77 patients with refractory AML and 52 patients with MDS were treated with conventional priming regimens (CHG or CAG). This was a single-center retrospective analysis of remission, adverse event, mortality, and survival. The capacity of clinical features (including the expression of co-stimulatory molecule B7.1 on tumor cells) to influence survival was assessed by multivariate Cox regression.

Results: Complete and partial remission rates (RRs) were significantly higher in AML patients treated with new regimens compared to conventional ones ( $68.2 \%$ vs $13.6 \%, P<0.05)$. Complete and partial remission were also significantly higher in patients with MDS treated with new regimens (55.6\% vs $19.4 \%, P<0.05$ ). However, although survival advantages were observed in the first year, the new regimens did not significantly improve 3-year overall survival $(P>0.05)$. Patients administered the new regimens experienced more severe and sustained myelosuppression $(P<0.05)$, but no severe adverse events or treatment-related deaths were observed. The rate of non-hematological side effects did not differ significantly between treatment regimens $(P>0.05)$. Both RR and B7.1 expression were significantly higher in patients with AML-M2 and M5 $(P<0.05)$.

Conclusion: The new priming regimens improved the RR, lowered the recurrence rate, and improved survival in AML and middle-and-high-risk MDS, without significantly increasing adverse events.

Keywords: priming chemotherapy, acute myeloid leukemia, myelodysplastic syndrome, B7.1

\section{Introduction}

Refractory acute myeloid leukemia (AML) and middle-and-high-risk myelodysplastic syndrome (MDS; refractory anemia with excess blasts [RAEB] and refractory anemia with excess blasts in transformation [RAEBT]) are progressive clonal hematopoietic stem cell disorders associated with marrow dysplasia, ineffective hematopoiesis, and anemia. While chemotherapeutic regimen has demonstrated success in inducing remission, AML often progresses subsequently into refractory leukemia, and both AML and MDS are associated with diverse complications, short survival time, and poor long-term survival. ${ }^{1-7}$ 
Allogeneic hematopoietic stem cell transplantation (HSCT) is the most effective therapy for MDS and AML; however, as these diseases occur predominantly in older patients with a high rate of comorbidities, ${ }^{8,9}$ these patients have a low tolerance for both allogeneic HSCT and chemotherapy. ${ }^{1-9}$ Although high-dose chemotherapy regimens including fludarabine, cytarabine (AraC), granulocyte colony-stimulating factor (G-CSF), and HSCT are helpful for some young patients, the transplant efficacy is low, myelosuppression is severe and persistent, and morbidities like severe infection and mortality are high, especially in those with advanced age, hypocellular leukemia (HLA), secondary leukemia, and high-risk MDS.

Nevertheless, low-dose priming regimens including AraC or aclarubicin (Acla) with G-CSF (CAG regimen) have been reported to be safe and effective and to cause few adverse events. ${ }^{10-17} \mathrm{G}-\mathrm{CSF}$ is thought to increase the fraction of leukemic cells in S-phase, allowing chemotherapeutics AraC and Acla, which target this phase of cell cycle, to induce tumor cell cytotoxicity. However, despite improved remission rates (RRs), long-term survival remains poor, and the cardiac toxicity associated with Acla limits its application in elderly patients with preexisting cardiac comorbidities.

The cephalotaxus plant alkaloid homoharringtonine (HHT) was reported to cause arrest of leukemic cell cycle, inducing apoptosis, ${ }^{18,19}$ and has been used in the treatment of AML in People's Republic of China for decades ${ }^{20}$ in combination with G-CSF and AraC priming (CHG regimen). ${ }^{21-28}$ As HHT arrests cell cycle at a different phase from AraC, these drugs are hypothesized to act synergistically..$^{29,30}$

Here, we conducted a single-center retrospective study to assess the efficacy of new synergism-based, dose-enhanced, combined priming regimens in the treatment of refractory AML and middle-and-high-risk MDS. We compared the outcomes of patients treated with conventional CAG and CHG regimens with those treated with new regimens including the CHAG regimen (AraC, HHT, Acla, and G-CSF), CHTG regimen (AraC, HHT, pirarubicin (THP), and G-CSF), CHMG regimen (AraC, HHT, mitoxantrone, and G-CSF), and CTMG regimen (AraC, THP, mitoxantrone, and G-CSF), and aimed to identify demographic and clinical patient characteristics associated with outcomes.

\section{Materials and methods \\ Patients}

This non-randomized, single-center, retrospective cohort trial was designed and conducted at the Hematology Department of the Second Affiliated Hospital of Xi'an Jiaotong
University. Under the approval of Medical Academic Board and Institutional Review Board, patients with either refractory AML $(\mathrm{n}=121)$ or middle-and-high-risk MDS $(\mathrm{n}=88)$ were treated between January 2004 and June 2014. Written informed consent was provided by all patients and/or their legal guardians.

Inclusion criteria included diagnosis with either AML or MDS according to the morphologic, immunophenotypic, cytogenetic, and molecular protocols of the Diagnostic and Therapeutic Criteria for Hematological Diseases, ${ }^{31}$ consistent with the Second National Refractory Leukemia Seminar criteria, NCCN \& ESMO Clinical Practice Guidelines, Guidelines on the Management of AML in Chinese Adults, and Chinese Expert Consensus Guidelines for the Diagnosis and Treatment of MDS.

AML was categorized according to French-AmericanBritish classification, ${ }^{32}$ or as recurrent $(n=47)$, drug-resistant AML ( $\mathrm{n}=32)$, HLA $(\mathrm{n}=28)$, and secondary/transformed leukemia $(\mathrm{n}=14)$. MDS was categorized as either RAEB or RAEBT.

Baseline demographic and clinical criteria and Eastern Cooperative Oncology Group scores were determined at the time of treatment. A subset of patients was further examined by karyotyping and leukemia fusion gene analysis.

\section{Study design}

The outcome of patients receiving conventional priming regimens ( $\mathrm{CHG}$ and $\mathrm{CAG}$ ) was compared to that of patients receiving new priming regimens (CHAG, $\mathrm{CHTG}, \mathrm{CHMG}$, and CTMG). Complete remission (CR), partial remission (PR), and nonremission were defined according to the Diagnostic and Therapeutic Criteria for Hematological Diseases, the Second National Refractory Leukemia Seminar criteria, and the International Workshop on Leukemia guidelines. RR was calculated as $\mathrm{CR}+\mathrm{PR}$. A regimen would be repeated when it achieved PR. The efficacy of the regimen (CR, PR, and nonremission) was evaluated by bone marrow morphology assessment 2 weeks after the end of the regimen.

\section{Measurement of tumor cell expression of B7.I}

The expression of B7.1 on bone marrow cells was assessed by immunostaining and flow cytometry before initiation of chemotherapy. Briefly, fresh bone marrow samples $(2 \mathrm{~mL})$ were mixed with heparin for Ficoll-Hypaque gradient centrifugation. The mononuclear cell fraction was subsequently routinely washed with phosphate-buffered saline three times and resuspended in phosphate-buffered saline 
at $1 \times 10^{6} / \mathrm{mL}$. M-fluorescein isothiocyanate-conjugated mouse anti-human cluster of differentiation 80 antibody (Biosciences Pharmingen, San Jose, CA, USA), was added to each sample, and staining was assessed by flow cytometry. A specimen was defined as positive if $\geq 20 \%$ of total cell count stained positive for B7.1, or otherwise as negative.

\section{Therapeutic regimens}

The conventional $\mathrm{CHG}$ priming regimen consisted of subcutaneous injection of $100 \mu \mathrm{g} / \mathrm{m}^{2}$ of G-CSF, intravenous drip of $1.0 \mathrm{mg} / \mathrm{m}^{2}$ HHT daily from d1 to 14 , and subcutaneous injection of $7.5-10 \mathrm{mg} / \mathrm{m}^{2}$ of AraC every 12 hours. G-CSF was administered from 2 hours before initiation of $\mathrm{AraC}$ to 12 hours before discontinuing the last dose of AraC. The conventional $\mathrm{CAG}$ priming regimen replicated the $\mathrm{CHG}$ regimen, but HHT was replaced with $12 \mathrm{mg} / \mathrm{m}^{2}$ of Acla, administered every other day. The new $\mathrm{CHAG}$ priming regimen combined the CAG and CHG regimen, and both HTT and Acla were administered as previously described. The new CHTG priming regimen replicated the $\mathrm{CHAG}$ regimen, but Acla was replaced with $12 \mathrm{mg} / \mathrm{m}^{2}$ of THP administered every other day. The new $\mathrm{CHMG}$ priming regimen replicated the $\mathrm{CHAG}$ priming regimen, but Acla was replaced with $2 \mathrm{mg} / \mathrm{m}^{2}$ of mitoxantrone every other day. The new CTMG priming regimen replicated the CHTG priming regimen, but HTT was replaced with mitoxantrone.

The following symptomatic and supportive treatments were also used. G-CSF was continued after the course of chemotherapy if the peripheral white blood cell (WBC) count fell $<20 \times 10^{9} / \mathrm{L}$ until rebound at $>20 \times 10^{9} / \mathrm{L}$. Recombinant human thrombopoietin and hemostatic therapy were administered where platelet (PLT) count fell $<50 \times 10^{9} / \mathrm{L}$, and PLT suspension was injected if PLT count fell $<20 \times 10^{9} / \mathrm{L}$ or the patient exhibited hemorrhagic tendency. Erythrocyte suspension was injected to those with $<60 \mathrm{~g} / \mathrm{L}$ hemoglobin, poor cardiac/pulmonary compensation, or self-reported symptoms of ischemia. If grade IV myelosuppression occurred, patients were transported to a sterile laminar flow ward, and G-CSF was administered in addition to antibiotics, antifungals, and immune supportive therapy.

Where PR was achieved, the regimen was not assessed until 2 weeks after completion of the next course of treatment. Responsive patients were administered six to eight courses of maintenance therapy composed of the primary regimen, standard DA (soft erythromycin, cytarabine) regimen, and median dose-AraC regimen, while nonresponsive patients were eligible for this trial. Chemotherapy dose was reduced by $25 \%$ if grade IV (life-threatening) toxicity occurred.

\section{Outcome analysis}

Clinical manifestations, adverse events, electrocardiogram, bone marrow biopsy, bone marrow B7.1 expression, routine blood tests for liver and kidney functions, and cardiac enzymes were assessed pre- and post-chemotherapy. Bone marrow samples were taken, and minimal residual disease was assessed 1-2 weeks after chemotherapy was completed. Short-term curative effects were assessed after two courses of treatment.

Patients were monitored using weekly routine blood tests from the initiation of chemotherapy, and after each course of chemotherapy, bone marrow biopsy and minimal residual disease examination were performed.

Patients were followed up until December 31, 2014, with a mean follow-up ranging from 6 to 66.9 months (median, 37.2 months; shortest follow-up, 3 months). Nineteen patients (9\%) were lost to follow-up. Overall survival (OS) was calculated, and survival curve plotted.

\section{Adverse event recording}

The toxicity of chemotherapy was classified into 0 (none), I (mild), II (moderate), III (severe), and IV (life-threatening) based on the World Health Organization toxicity classification system and tagged according to the Common Terminology Criteria for Adverse Events Version 4.0 developed by the National Cancer Institute.

\section{Statistical analysis}

Data were analyzed using SPSS 18.0 (IBM, Armonk, NY, USA). Continuous data were compared using the independentsamples $t$-test or the Mann-Whitney nonparametric test, as appropriate. Frequencies of each group were compared by Pearson's chi-squared test or continuous-corrected chi-squared test. Survival rate was estimated by the Kaplan-Meier method and compared using the log-rank test. The capacity of clinical features to influence outcome and survival was assessed by multivariate cox regression. $P$-value was derived from twotailed tests, with $P<0.05$ defined as significant difference.

\section{Results \\ Patient demographic and clinical characteristics}

A total of 209 patients with either refractory AML $(n=121)$ or middle-and-high-risk MDS $(n=88)$ were enrolled in this non-randomized, single-center, retrospective cohort study (Table 1). The outcome of patients receiving conventional priming regimens ( $\mathrm{CHG}$ and $\mathrm{CAG}$ ) was compared to that of patients receiving the new priming regimens (CHAG, $\mathrm{CHTG}$, CHMG, and CTMG). 
Table I Patient demographic and clinical characteristics

\begin{tabular}{|c|c|c|c|}
\hline Variables & New priming regimens $(n=80)$ & Conventional priming regimens $(n=129)$ & $P$-value \\
\hline Age, median (range) & $54(|5-8|)$ & $50(14-78)$ & 0.878 \\
\hline \multicolumn{4}{|l|}{ Sex, n (\%) } \\
\hline Male & 42 & 69 & 0.889 \\
\hline Female & 38 & 60 & \\
\hline \multicolumn{4}{|l|}{ Disease subtype } \\
\hline AML & 44 & 77 & 0.504 \\
\hline MI & 4 & 10 & 0.439 \\
\hline M2 & 17 & 28 & 0.938 \\
\hline M4 & 7 & 17 & 0.329 \\
\hline M5 & 16 & 22 & 0.591 \\
\hline MDS & 36 & 52 & 0.504 \\
\hline RAEB & 26 & 34 & 0.340 \\
\hline RAEBT & 10 & 18 & 0.764 \\
\hline \multicolumn{4}{|l|}{ Risk stratification } \\
\hline Low & 5 & 8 & 1.000 \\
\hline Middle & 42 & 66 & 0.851 \\
\hline High & 33 & 55 & 0.844 \\
\hline WBC count $\left(\times 10^{9} / L\right)$ & $8.77(0.6-30)$ & $9.15(0.4-32)$ & 0.449 \\
\hline RBC count $\left(\times 10^{12} / \mathrm{L}\right)$ & $2.1(1.0-5.1)$ & $2.3(1.3-4.9)$ & 0.965 \\
\hline PLT count $\left(\times 10^{9} / \mathrm{L}\right)$ & $34(2-121)$ & $28(\mathrm{I}-10 \mathrm{I})$ & 0.898 \\
\hline Myeloblast (\%) & 31.17 (5.5-93) & $34.14(5.5-81)$ & 0.565 \\
\hline Karyotype t $(8 ; 21)$ & 26 & 38 & 0.643 \\
\hline
\end{tabular}

Abbreviations: AML, acute myeloid leukemia; MDS, myelodysplastic syndrome; RAEB, refractory anemia with excess blasts; RAEBT, refractory anemia with excess blasts in transformation; WBC, white blood cell; RBC, red blood cell; PLT, platelet.

The patient group included 111 males and 98 females with a median age of 51 (14-81) years. Patients with AML were classified according to the French-American-British classification $^{32}$ as M1 ( $\left.\mathrm{n}=14\right)$, M2 $(\mathrm{n}=45), \mathrm{M} 4(\mathrm{n}=24)$, and M5 ( $n=38)$, or with recurrent ( $n=47)$, drug-resistant AML $(\mathrm{n}=32)$, HLA $(\mathrm{n}=28)$, and secondary/transformed leukemia $(\mathrm{n}=14)$. The MDS group included 60 patients with RAEB and 28 patients with RAEBT.

At diagnosis, the peripheral WBC count ranged from 0.4 to $70 \times 10^{9} / \mathrm{L}$, red blood cell count from 1.0 to $5.1 \times 10^{12} / \mathrm{L}$, PLT count from 1 to $121 \times 10^{9} / \mathrm{L}$, and myeloblast percentage from $5.5 \%$ to $93 \%$. Patients were further examined by karyotyping and leukemia fusion gene analysis. The rate of the advantageous karyotype $t(8 ; 21)$ did not differ significantly between the groups.

Forty-four patients with AML and 36 patients with MDS received new priming regimens, and 77 patients with AML and 52 patients with MDS received conventional priming regimens. The demographic and clinical characteristics of the patients assigned to the new priming regimens $(n=80)$ did not differ significantly from those assigned to the conventional priming regimens $(\mathrm{n}=129)(P>0.05$; Table 1$)$, and they had similar Eastern Cooperative Oncology Group scores before treatment. No patients required dose adjustment due to liver or kidney dysfunction.

\section{B7.I expression on tumor cells}

The rate of bone marrow blast B7.1 expression was significantly higher in patients with AML (51.2\%) than in patients with MDS (10.2\%), and was significantly higher in patients with AML-M2 (64.4\%) or AML-M5 (65.8\%) than in the other AML subgroups (all $P<0.05$ ). For individual patients, B7.1 expression was positively correlated with the efficacy of priming regimen. In other words, most patients responsive to chemotherapy were exhibiting bone marrow blast B7.1 expression, whereas B7.1-negative patients rarely responded to any treatments (Table 2).

Table 2 B7.I expression in AML and MDS subgroups

\begin{tabular}{cll}
\hline Group & $\mathbf{N}$ & B7.I expression (\%) \\
\hline AML & 12 I & $62(51.24 \%)$ \\
MI & 14 & $3(21.43 \%)$ \\
M2 & 45 & $29(64.44 \%)^{\mathrm{a}}$ \\
M4 & 24 & $5(20.83 \%)$ \\
M5 & 38 & $25(65.79 \%)^{\mathrm{b}}$ \\
MDS & 88 & $9(10.23 \%)$ \\
RAEB & 60 & $5(8.33 \%)$ \\
RAEBT & 28 & $4(14.28 \%)$ \\
\hline
\end{tabular}

Notes: ${ }^{a}<0.05$ vs other AML subtype. ${ }^{b}<0.05$ vs MDS subtype.

Abbreviations: AML, acute myeloid leukemia; MDS, myelodysplastic syndrome; RAEB, refractory anemia with excess blasts; RAEBT, refractory anemia with excess blasts in transformation. 

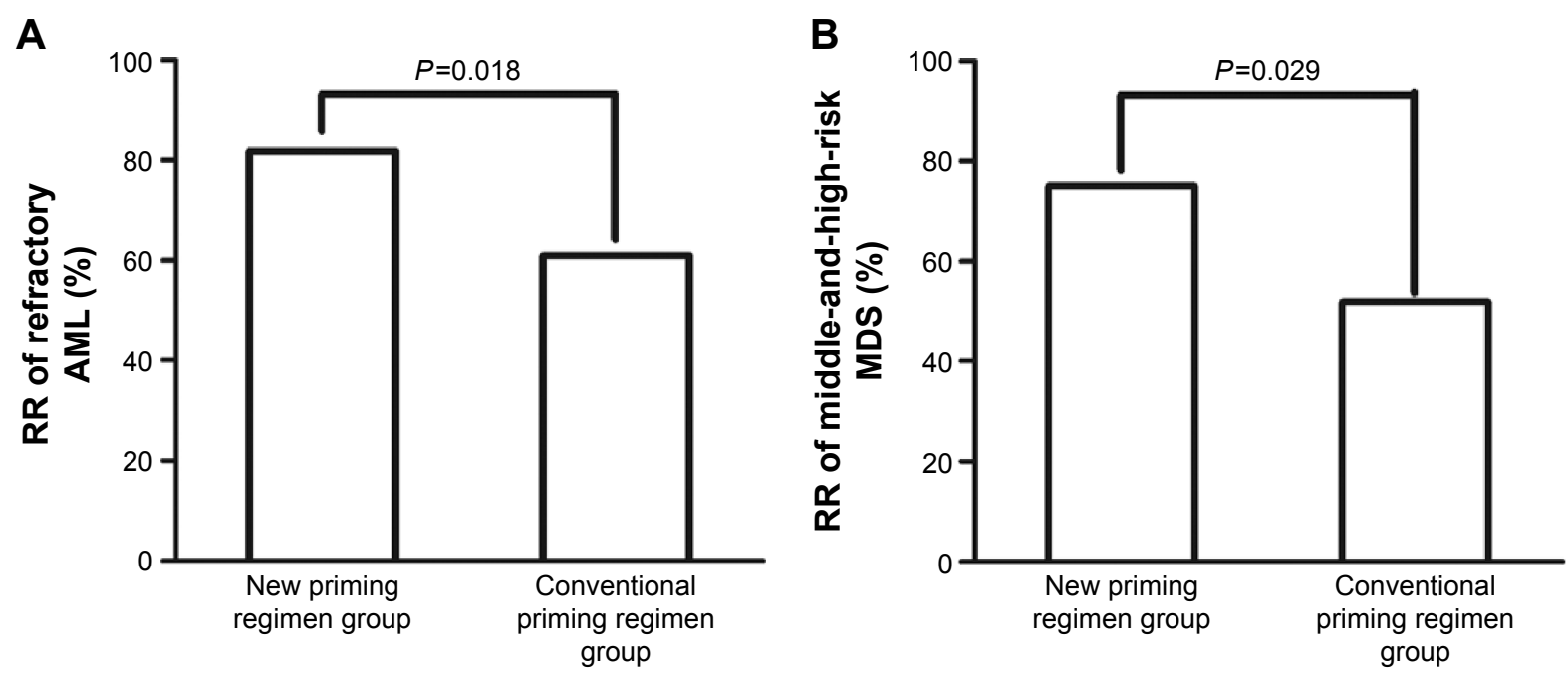

Figure I Comparison of the response rate in refractory AML $(\mathbf{A})$ and middle-and-high-risk MDS $(\mathbf{B})$ patients administered new and conventional priming regimens. Abbreviations: RR, remission rate; AML, acute myeloid leukemia; MDS, myelodysplastic syndrome.

\section{Short-term efficacy}

Response rates were assessed after two courses of chemotherapy. In patients with refractory AML, the RR and CR and $\mathrm{PR}$ rates were significantly higher in patients administered the new priming regimens (36/44 [81.8\%], 30/44 [68.2\%], and $6 / 44$ [13.6\%], respectively) than in patients administered conventional priming regimens (47/77 [61.0\%], 40/77 [52.0\%], and 7/77 [9.1\%], respectively) (RR, $P=0.018$ ) (Figures $1 \mathrm{~A}$, $2 \mathrm{~A}$, and $3 \mathrm{~A})$. The rates in the AML-M2 (15/17) and AML-M5 $(14 / 16)$ subgroups were significantly higher than in the other AML subgroups $(P<0.05)$.
Similarly, in patients with middle-and-high-risk MDS, the $\mathrm{RR}$ and $\mathrm{CR}$ and $\mathrm{PR}$ rates were significantly higher in patients administered the new priming regimens (27/36 [75.0\%], $20 / 36$ [55.6\%], and 7/36 [19.4\%], respectively) than in patients administered conventional priming regimens $(27 / 52$ [51.9\%], 19/52 [36.5\%], and 8/52 [15.4\%]) $(\mathrm{RR}, P=0.029)$ (Figures 1B, 2B, and 3B).

Subgroup analysis revealed that patients younger than 70 years had a slightly but not significantly higher RR than those $>70$ years $(80.0 \%$ vs $76.7 \%, P=0.787)$. Patients with $>30 \%$ myeloblasts had a slightly but not significantly
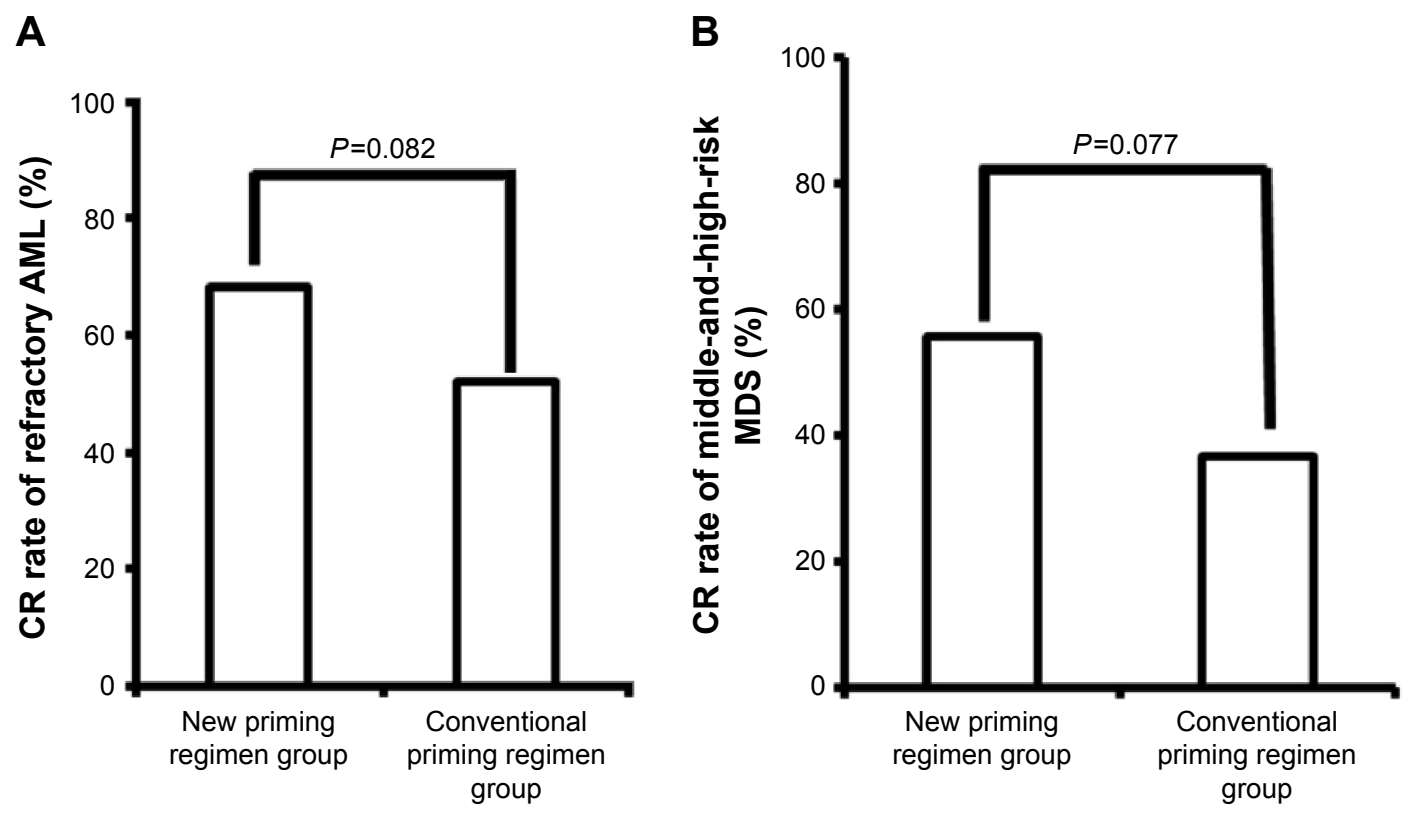

Figure 2 Comparison of the complete remission rate in refractory AML (A) and middle-and-high-risk MDS (B) patients administered new and conventional priming regimens.

Abbreviations: CR, complete remission; AML, acute myeloid leukemia; MDS, myelodysplastic syndrome. 
A

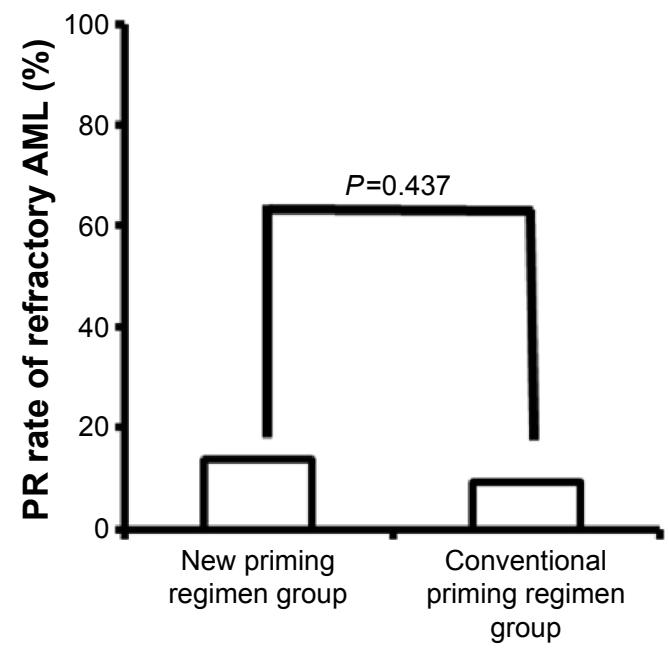

B

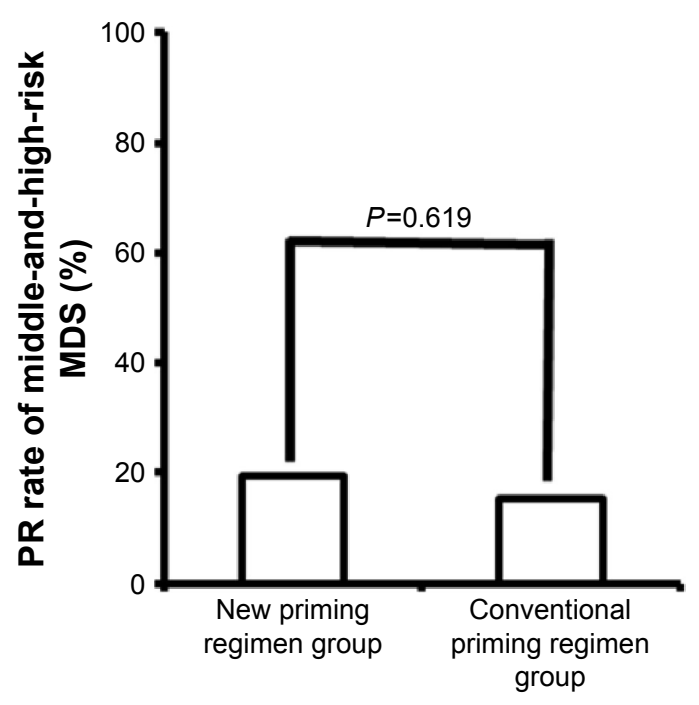

Figure 3 Comparison of the partial remission rates in refractory AML (A) and middle-and-high-risk MDS (B) patients administered new and conventional priming regimens. Abbreviations: PR, partial remission; AML, acute myeloid leukemia; MDS, myelodysplastic syndrome.

higher RR (80.6\% vs 77.3\%, $P=0.685)$. However, the RR was significantly higher in patients with advantageous karyotype $t(8 ; 21)$ than those without, or with other complex karyotypes ( $92.3 \%$ vs $72.2 \%, P=0.040)$.

\section{Long-term survival analyses}

Patients were followed up until December 31, 2014 (median, 36.2 months; shortest follow-up, 3 months), and 19 (9\%) patients were lost to follow-up. The 1-, 2-, and 3-year OS did not differ significantly between AML patients in the new and conventional priming regimen groups $(79.5 \%$ vs $62.3 \%, P=0.050 ; 40.9 \%$ vs $32.5 \%$, $P=0.351 ; 18.2 \%$ vs $15.6 \%, P=0.711$, respectively), but a difference was observed for the 1-year OS in patients with MDS between the new and conventional priming regimens, but not for 2- and 3-year OS (69.4\% vs 48.1\%, $P=0.047 ; 33.3 \%$ vs $21.2 \%, P=0.201 ; 11.1 \%$ vs $9.6 \%$, $P=1.000$, respectively), indicating that the new priming regimens did not significantly improve the 3-year OS $(P>0.05)$, although survival advantages were observed in year 1 (Figure 4 ).

\section{Cox survival analysis}

To determine the clinical predictors of outcome, multivariate Cox regression was used. The results showed that the complex karyotype was an independent prognostic factor for survival (hazard ratio 4.427, 95\% confidence interval 2.687-7.295), while chemotherapy regimen, WBC, and rate of bone marrow blast B7.1 expression were not (Table 3).
A

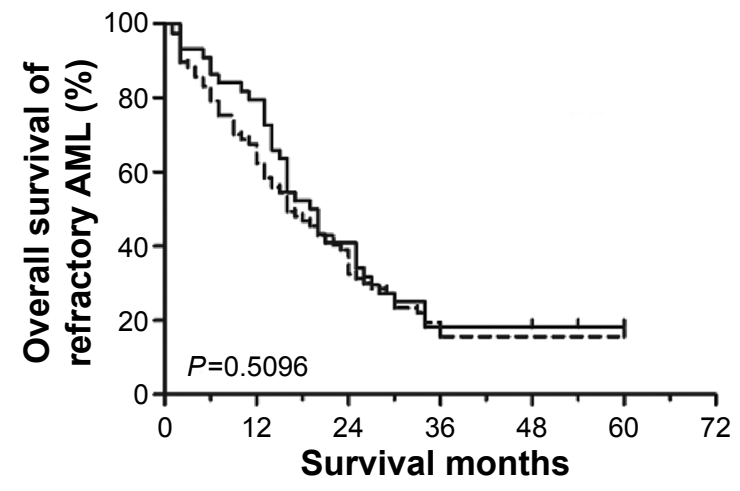

B

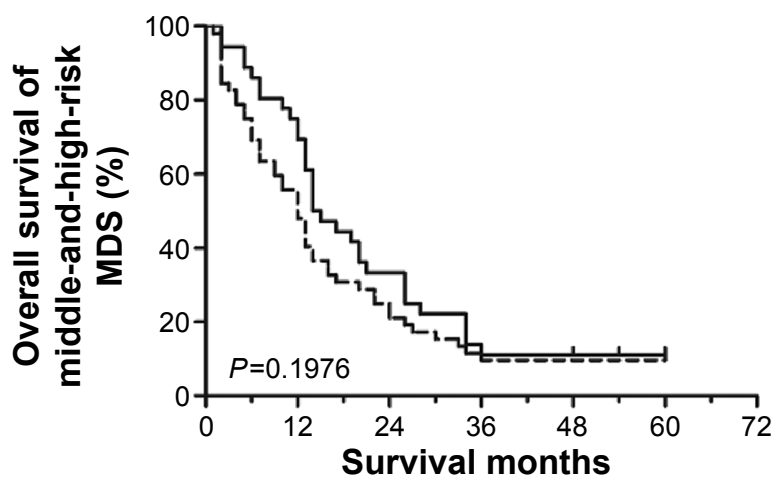

ـ New priming regimen group ㄴ. Conventional priming regimen group

Figure 4 Comparison of long-term OS in refractory AML (A) and middle-and-high-risk MDS (B) patients administered the new and conventional priming regimen. Abbreviations: AML, acute myeloid leukemia; MDS, myelodysplastic syndrome; OS, overall survival. 
Table 3 Cox survival analysis

\begin{tabular}{lllll}
\hline Risk factors & P-value & HR & \multicolumn{2}{c}{$\mathbf{9 5 \%} \mathbf{C l}$} \\
\cline { 4 - 5 } & & & Lower & Upper \\
\hline Priming chemotherapy & 0.058 & 1.647 & 0.983 & 2.76 \\
White blood cell counts & 0.438 & 1.024 & 0.965 & 1.086 \\
Bone marrow blast cell percentage & 0.906 & 0.999 & 0.976 & 1.022 \\
Complex karyotype & 0 & 4.427 & 2.687 & 7.295 \\
\hline
\end{tabular}

Abbreviations: $\mathrm{HR}$, hazard ratio; $\mathrm{Cl}$, confidence interval.

\section{Safety and toxicity}

Within the 4 weeks following the completion of chemotherapy (mean duration, 5-26 days), adverse events such as agranulocytosis, thrombocytopenia, and anemia were recorded. AML and MDS patients administered new priming regimens suffered from more severe and sustained myelosuppression than patients administered the conventional priming regimens $(P<0.05)$; however, no severe hemorrhage, infection, or treatment-related death was observed in this sample. The rate of non-hematologic toxicities such as gastrointestinal, cardiac, hepatic, or renal dysfunction did not differ significantly between patients administered new or conventional priming regimens $(P>0.05)$, and these dysfunctions resolved with timely proactive interventions (Table 4).

\section{Discussion}

In this single-center retrospective study, we compared the efficacy of conventional and novel chemotherapeutic regimens for the treatment of patients with refractory AML or middle-andhigh-risk MDS. The established low-dose chemotherapeutic regimens $\mathrm{CHG}$ and CAG have been reported to be safe and effective, and to cause few adverse events, ${ }^{10-17}$ though the rate of cardiac toxicity associated with Acla limits its application in elderly patients and long-term outcomes remain poor.

In this study, we found that more patients with AML and MDS treated with the new chemotherapeutic regimens (CHAG, CHTG, CHMG, and CTMG) achieved CR and PR compared to AML patients treated with conventional regimens. The new regimens were associated with survival advantages in the first year, but they did not significantly improve 3-year OS. In addition, patients administered new regimens experienced more severe and sustained myelosuppression, but no severe adverse events or treatment-related death was observed, and the rate of non-hematologic side effects did not differ significantly between treatment regimens. All the adverse effects were successfully treated after proactive intervention, suggesting that the severe adverse effects were not increased while the dose was increased, finally resulting in improved benefits for the patients.

A previous study reported that a GHA priming regimen containing G-CSF, HHT, and low-dose AraC was effective for relapsed/refractory AML and middle-and-high-risk MDS with excess blasts. ${ }^{33}$ Pre-chemotherapy administration of G-CSF is thought to drive G0-phase cells into the

Table 4 Adverse events (grade III/IV)

\begin{tabular}{|c|c|c|c|c|c|c|}
\hline Variables & $\begin{array}{l}\text { New priming } \\
\text { regimens - AML } \\
(\mathrm{N}=44)\end{array}$ & $\begin{array}{l}\text { Conventional priming } \\
\text { regimens - AML } \\
(\mathbf{N}=77)\end{array}$ & $P$-value & $\begin{array}{l}\text { New priming } \\
\text { regimens - MDS } \\
(\mathrm{N}=36)\end{array}$ & $\begin{array}{l}\text { Conventional priming } \\
\text { regimens - MDS } \\
(\mathrm{N}=52)\end{array}$ & P-value \\
\hline Leukopenia & 39 (88.6\%) & $45(58.4 \%)$ & 0.001 & $32(88.9 \%)$ & $29(55.8 \%)$ & 0.001 \\
\hline Anemia & $36(81.8 \%)$ & 49 (63.6\%) & 0.035 & 32 (88.9\%) & $32(61.5 \%)$ & 0.005 \\
\hline Thrombocytopenia & 40 (90.9\%) & $53(68.8 \%)$ & 0.006 & $31(86.1 \%)$ & $33(63.5 \%)$ & 0.019 \\
\hline Time to reach $>20 \times 10^{9} / \mathrm{L}$ (days) & $\mid 4(7-2 \mid)$ & $8(6-12)$ & & II (8-18) & $7(5-11)$ & \\
\hline Neutropenia & $36(81.8 \%)$ & $45(58.4 \%)$ & 0.009 & $29(80.6 \%)$ & $28(53.9 \%)$ & 0.010 \\
\hline Time to reach $>0.5 \times 10^{9} / \mathrm{L}$ (days) & $17(8-26)$ & $9(5-14)$ & & $14(6-2 \mid)$ & $8(6-13)$ & \\
\hline Nausea, vomiting & $6(13.6 \%)$ & $8(10.4 \%)$ & 0.591 & $4(11.1 \%)$ & $5(9.6 \%)$ & 1.000 \\
\hline Liver dysfunction & $2(4.5 \%)$ & $\mathrm{I}(1.3 \%)$ & 0.619 & I (2.8\%) & $\mathrm{I}(1.9 \%)$ & 1.000 \\
\hline Kidney dysfunction & I $(2.3 \%)$ & 0 & 0.776 & 0 & 0 & - \\
\hline Alopecia & $3(6.8 \%)$ & I (I.3\%) & 0.269 & I $(2.8 \%)$ & 0 & 0.852 \\
\hline Cardiac injury & 0 & 0 & - & 0 & 0 & - \\
\hline Oral ulcer & $10(22.7 \%)$ & 15 (19.5\%) & 0.671 & $6(16.7 \%)$ & 7 (13.5\%) & 0.677 \\
\hline Neutropenic fever & $25(56.8 \%)$ & $36(46.8 \%)$ & 0.287 & $20(55.6 \%)$ & 25 (48.1\%) & 0.490 \\
\hline RBC transfusion (patients) & 40 (90.9\%) & 55 (7I.4\%) & 0.012 & 34 (94.4\%) & 35 (67.3\%) & 0.003 \\
\hline Cell cycle & $38(86.4 \%)$ & $51(66.2 \%)$ & 0.016 & 33 (91.7\%) & $33(63.5 \%)$ & 0.003 \\
\hline PLT transfusion (patients) & $4 \mathrm{I}(93.2 \%)$ & $54(70.1 \%)$ & 0.003 & 33 (91.7\%) & 35 (67.3\%) & 0.007 \\
\hline Cell cycle & 40 (90.9\%) & $52(67.5 \%)$ & 0.004 & $30(83.3 \%)$ & 31 (59.6\%) & 0.018 \\
\hline Antibiotic use (patients) & 39 (88.6\%) & $56(72.7 \%)$ & 0.040 & $28(77.8 \%)$ & 31 (59.6\%) & 0.075 \\
\hline Cell cycle & $34(77.3 \%)$ & 45 (58.4\%) & 0.091 & $25(69.4 \%)$ & $28(53.8 \%)$ & 0.142 \\
\hline
\end{tabular}

Note: Data in bold indicates statistical significance $(P<0.05)$.

Abbreviations: AML, acute myeloid leukemia; MDS, myelodysplastic syndrome; RBC, red blood cell; PLT, platelet. 
proliferative phase and thus promotes chemosensitivity. Intracellular ATP level is elevated, enhancing the cytotoxic effect of AraC on leukemic cells. ${ }^{33}$ Low-concentration decitabine has been reported to demethylate a key transcription factor of myeloid differentiation in vitro, and to induce cell maturity and remission of leukemia; however, the clinical efficacy of decitabine alone remains low. A priming regimen combining decitabine with low-dose AraC was thus hypothesized to improve efficacy, though the optimal administration sequence and required dosage remain to be determined. A more effective, lowly toxic, applicable, localized, tolerable, and economical combined priming regimen based on GHA regimen should be developed for relapsed/refractory AML and middle-and-high-risk MDS with excess blasts.

Liu et al reported that $\mathrm{CAG}$ regimens containing Acla $5-7 \mathrm{mg} / \mathrm{m}^{2}$ for 14 days resulted in a significantly higher $\mathrm{RR}$ than conventional priming regimens without increasing adverse events, indicating that an appropriately increased dose may effectively maximize the therapeutic effects. ${ }^{15}$ In order to maximize drug synergism and minimize drug resistance, we assessed the clinical outcomes associated with four different priming regimens and found that the novel regimens performed better than conventional ones.

Chen et $\mathrm{al}^{34}$ also reported a higher RR, better tolerability, and more survival advantages using modified CHAG priming regimens, probably owing to drug synergism of combined priming regimens. We compared the therapeutic efficacy between different subtypes of AML and MDS and reported for the first time significantly higher effective rates in patients with AML-M2 and M5. Additionally, Cox analysis indicated that complex karyotypes were an independent prognostic indicator of longer survival, indicating that the assessment of prognostic factors and treatment stratification may allow new priming regimen to be tailored to the disease profile to improve efficacy.

Tumor cells may evade host immunosurveillance by inactivating $\mathrm{T}$ cell-mediated specific antitumor immune responses by limiting expression of tumor-specific antigens or depressing expression of major histocompatibility complex or co-stimulatory molecules (especially B7.1). ${ }^{35-38}$ In our study, levels of blast B7.1 expression differed significantly between patients with AML and MDS, and between patient subtypes. The patients with AML-M2 and M5 exhibited significantly higher levels of B7.1 expression than the other AML subgroups, and B7.1 expression was positively associated with the efficacy of individual priming regimen. This association and the mechanisms underlying it should be further validated in a larger patient group. As this study included a limited number of patients at a single site, the results will require validation using larger prospective multicenter randomized controlled trials. The potential improvement associated with individual regimen could also be directly compared in a larger sample.

\section{Acknowledgment}

This work was supported by the 2013 Science and Technology Research and Development Project of Shanxi Province (No 2013K12-06-03).

\section{Disclosure}

The authors report no conflicts of interest in this work.

\section{References}

1. Hoyle CF, de Bastos M, Wheatley K, et al. AML associated with previous cytotoxic therapy, MDS or myeloproliferative disorders: results from the MRC's 9th AML trial. Br J Haematol. 1989;72(1):45-53.

2. Oosterveld M, Muus P, Suciu S, et al. Chemotherapy only compared to chemotherapy followed by transplantation in high risk myelodysplastic syndrome and secondary acute myeloid leukemia; two parallel studies adjusted for various prognostic factors. Leukemia. 2002;16(9):1615-1621.

3. Greenberg P, Cox C, LeBeau MM, et al. International scoring system for evaluating prognosis in myelodysplastic syndromes. Blood. 1997; 89(6):2079-2088.

4. Menzin J, Lang K, Earle CC, Kerney D, Mallick R. The outcomes and costs of acute myeloid leukemia among the elderly. Arch Intern Med. 2002; 162(14):1597-1603.

5. Oran B, Weisdorf DJ. Survival for older patients with acute myeloid leukemia: a population-based study. Haematologica. 2012;97(12): 1916-1924.

6. Buchner T, Berdel WE, Haferlach C, et al. Age-related risk profile and chemotherapy dose response in acute myeloid leukemia: a study by the German Acute Myeloid Leukemia Cooperative Group. J Clin Oncol. 2009;27(1):61-69.

7. Rollig C, Thiede C, Gramatzki M, et al. A novel prognostic model in elderly patients with acute myeloid leukemia: results of 909 patients entered into the prospective AML96 trial. Blood. 2010;116(6): 971-978.

8. Pulsoni A, Pagano L, Latagliata R, et al. Survival of elderly patients with acute myeloid leukemia. Haematologica. 2004;89(3):296-302.

9. Juliusson G, Antunovic P, Derolf A, et al. Age and acute myeloid leukemia: real world data on decision to treat and outcomes from the Swedish Acute Leukemia Registry. Blood. 2009;113(18):4179-4187.

10. Saito K, Nakamura Y, Aoyagi M, et al. Low-dose cytarabine and aclarubicin in combination with granulocyte colony-stimulating factor (CAG regimen) for previously treated patients with relapsed or primary resistant acute myelogenous leukemia (AML) and previously untreated elderly patients with AML, secondary AML, and refractory anemia with excess blasts in transformation. Int J Hematol. 2000;71(3):238-244.

11. Qian SX, Li JY, Tian T, et al. Effect of low-dose cytarabine and aclarubicin in combination with granulocyte colony-stimulating factor priming (CAG regimen) on the outcome of elderly patients with acute myeloid leukemia. Leuk Res. 2007;31(10):1383-1388.

12. Suzushima H, Wada N, Yamasaki H, et al. Low-dose cytarabine and aclarubicin in combination with granulocyte colony-stimulating factor for elderly patients with previously untreated acute myeloid leukemia. Leuk Res. 2010;34(5):610-614. 
13. Yamada K, Furusawa S, Saito K, et al. Concurrent use of granulocyte colony-stimulating factor with low-dose cytosine arabinoside and aclarubicin for previously treated acute myelogenous leukemia: a pilot study. Leukemia. 1995;9(1):10-14.

14. Li JM, Shen Y, Wu DP, et al. Aclarubicin and low-dose cytosine arabinoside in combination with granulocyte colony-stimulating factor in treating acute myeloid leukemia patients with relapsed or refractory disease and myelodysplastic syndrome: a multicenter study of 112 Chinese patients. Int J Hematol. 2005;82(1):48-54.

15. Liu L, Zhang Y, Jin Z, et al. Increasing the dose of aclarubicin in low-dose cytarabine and aclarubicin in combination with granulocyte colony-stimulating factor (CAG regimen) can safely and effectively treat relapsed or refractory acute myeloid leukemia. Int $J$ Hematol. 2014;99(5):603-608.

16. Fukushima T, Kawabata H, Sawaki T, et al. Low-dose cytarabine plus aclarubicin for patients with previously untreated acute myeloid leukemia or high-risk myelodysplastic syndrome ineligible for standard-dose cytarabine plus anthracycline. Anticancer Res 2012;32(4):1347-1353.

17. Wei G, Ni W, Chiao JW, Cai Z, Huang H, Liu D. A meta-analysis of CAG (cytarabine, aclarubicin, G-CSF) regimen for the treatment of 1,029 patients with acute myeloid leukemia and myelodysplastic syndrome. J Hematol Oncol. 2011;4:46

18. Wang J, Lu S, Yang J, et al. A homoharringtonine-based induction regimen for the treatment of elderly patients with acute myeloid leukemia: a single center experience from China. $J$ Hematol Oncol. 2009;2:32.

19. Mai WY, Lin MF. Induction of apoptosis by homoharringtonine in G1 phase human chronic myeloid leukemic cells. Chin Med J (Engl). 2005;118(6):487-492.

20. Kantarjian HM, Keating MJ, Walters RS, Koller CA, McCredie KB, Freireich EJ. Phase II study of low-dose continuous infusion homoharringtonine in refractory acute myelogenous leukemia. Cancer. 1989;63(5):813-817.

21. Jin J, Jiang DZ, Mai WY, et al. Homoharringtonine in combination with cytarabine and aclarubicin resulted in high complete remission rate after the first induction therapy in patients with de novo acute myeloid leukemia. Leukemia. 2006;20(8):1361-1367.

22. Wu L, Li X, Su J, et al. Efficacy and safety of CHG regimen (low-dose cytarabine, homoharringtonine with G-CSF priming) as induction chemotherapy for elderly patients with high-risk MDS or AML transformed from MDS. J Cancer Res Clin Oncol. 2011;137(10):1563-1569.

23. Wu L, Li X, Su J, et al. Effect of low-dose cytarabine, homoharringtonine and granulocyte colony-stimulating factor priming regimen on patients with advanced myelodysplastic syndrome or acute myeloid leukemia transformed from myelodysplastic syndrome. Leuk Lymphoma. 2009;50(9):1461-1467.
24. Smith CR Jr, Powell RG, Mikolajczak KL. The genus Cephalotaxus: source of homoharringtonine and related anticancer alkaloids. Cancer Treat Rep. 1976;60(8):1157-1170.

25. Cephalotaxus Research Coordinating Group. Cephalotaxine esters in the treatment of acute leukemia. A preliminary clinical assessment. Chin Med J (Engl). 1976;2(4):263-272.

26. Feldman E, Arlin Z, Ahmed T, et al. Homoharringtonine is safe and effective for patients with acute myelogenous leukemia. Leukemia. 1992;6(11):1185-1188.

27. Feldman E, Arlin Z, Ahmed T, et al. Homoharringtonine in combination with cytarabine for patients with acute myelogenous leukemia. Leukemia. 1992;6(11):1189-1191.

28. Feldman EJ, Seiter KP, Ahmed T, Baskind P, Arlin ZA. Homoharringtonine in patients with myelodysplastic syndrome (MDS) and MDS evolving to acute myeloid leukemia. Leukemia. 1996;10(1):40-42.

29. Lowenberg B, van Putten W, Theobald M, et al. Effect of priming with granulocyte colony-stimulating factor on the outcome of chemotherapy for acute myeloid leukemia. $N$ Engl J Med. 2003;349(8):743-752.

30. Kantarjian H, O'Brien S, Cortes J, et al. Results of intensive chemotherapy in 998 patients age 65 years or older with acute myeloid leukemia or high-risk myelodysplastic syndrome: predictive prognostic models for outcome. Cancer. 2006;106(5):1090-1098.

31. Zhang ZN, Shen T. Criteria for Diagnosis and Therapeutic Efficacy of Hematological Diseases 2007. 3rd ed. Beijing: Beijing Science Press; 2007.

32. Bennett JM, Catovsky D, Daniel MT, et al. Proposals for the classification of the acute leukaemias. French-American-British (FAB) co-operative group. Br J Haematol. 1976;33(4):451-458.

33. Jin J, Chen J, Suo S, et al. Low-dose cytarabine, aclarubicin and granulocyte colony-stimulating factor priming regimen versus idarubicin plus cytarabine regimen as induction therapy for older patients with acute myeloid leukemia. Leuk Lymphoma . 2015;56(6):1691-1697.

34. Chen L, Yin Q, Mi R, Wei X. CHAG priming regimen containing of cytarabine, aclacinomycin homoharringtonine and G-CSF for relapsed refractory acute myelogenous leukemia: a modified combination chemotherapeutic combination. Leuk Lymphoma. 2013;54(10): 2291-2293.

35. Greaves P, Gribben JG. The role of B7 family molecules in hematologic malignancy. Blood. 2013;121(5):734-744.

36. Sumimoto H, Tani K, Nakazaki Y, et al. GM-CSF and B7-1 (CD80) co-stimulatory signals co-operate in the induction of effective anti-tumor immunity in syngeneic mice. Int J Cancer. 1997;73(4):556-561.

37. Kaufman HL, Lenz HJ, Marshall J, et al. Combination chemotherapy and ALVAC-CEA/B7.1 vaccine in patients with metastatic colorectal cancer. Clin Cancer Res. 2008;14(15):4843-4849.

38. Zou W, Chen L. Inhibitory B7-family molecules in the tumour microenvironment. Nat Rev Immunol. 2008;8(6):467-477.
OncoTargets and Therapy

\section{Publish your work in this journal}

OncoTargets and Therapy is an international, peer-reviewed, open access journal focusing on the pathological basis of all cancers, potential targets for therapy and treatment protocols employed to improve the management of cancer patients. The journal also focuses on the impact of management programs and new therapeutic agents and protocols on

\section{Dovepress}

patient perspectives such as quality of life, adherence and satisfaction. The manuscript management system is completely online and includes a very quick and fair peer-review system, which is all easy to use. Visit http://www.dovepress.com/testimonials.php to read real quotes from published authors. 